
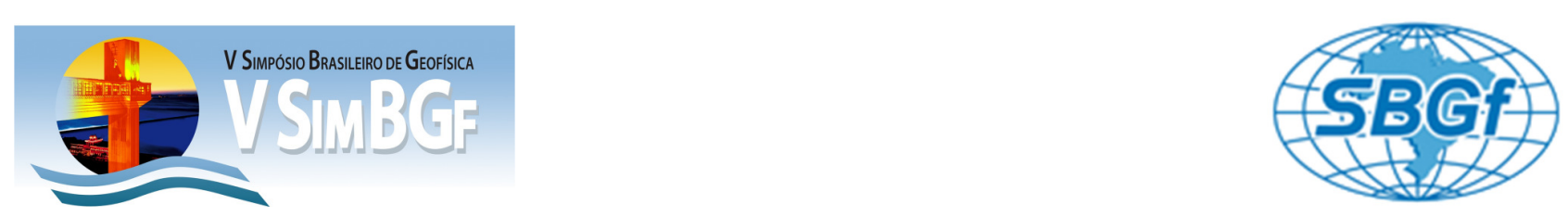

\title{
Gradiente Geotérmico nos bairros Jardim Primavera e São Geraldo II no Município de Montes Claros em Minas Gerais
}

Jéssica Luciano Barcelos (jessicalbarcelos@gmail.com) e Antônio Jorge de Lima Gomes (antonio.gomes@ufvim.edu.br) UFVJM - Universidade Federal dos Vales do Jequitinhonha e Mucuri

Copyright 2012, SBGf - Sociedade Brasileira de Geofísica

Este texto foi preparado para a apresentação no V Simpósio Brasileiro de Geofísica, Salvador, 27 a 29 de novembro de 2012. Seu conteúdo foi revisado pelo Comitê Técnico do V SimBGf, mas não necessariamente representa a opinião da SBGf ou de seus associados. É proibida a reprodução total ou parcial deste material para propósitos comerciais sem prévia autorização da SBGt.

\section{Resumo}

Results of updated compilations and geothermal investigations carried out during the period of 2010 to 2012 have allowed determination of geothermal gradients in districts Jardim Primavera e São Geraldo II, in the city of Montes Claros, State of Minas Gerais, Brazil. This new assessment is part of a research project called Gradient Flow and Geothermal Prospects with Exploitation of Geothermal Energy in the Valley of Jequitinhonha and Mucuri with support from FAPEMIG. The results were based on thermal temperature profiling performed on two wells undertaken by staff of the Geothermal Laboratory of the National Observatory/MCT of Rio de Janeiro, in 2005. The two wells are owned by COPASA and were built to supply water. The results obtained indicate few variation in geothermal gradients with values in the range of 18,6 and $20,2^{\circ} \mathrm{C} / \mathrm{km}$. The values of geothermal gradients in the two wells were obtained through the methodology of type CBT. This compilation of geothermal gradients used comparisons with different surface temperatures based on linear adjustments of the profiles at depths between 80 and 100 meters. Thus, the new values of the gradients ranged between 18.6 and $20.2{ }^{\circ} \mathrm{C} / \mathrm{km}$. These new values are considered as results corresponding to regions and related intracratonic sedimentary basins.

\section{Introdução}

Este trabalho tem como objetivo reavaliar os resultados dos gradientes geotérmicos em perfis de temperatura dos bairros Jardim Primavera e São Geraldo II no Município de Montes Claros, no Estado de Minas Gerais.

Os dados trabalhados foram obtidos durante um programa de pesquisa de Energia Geotérmica de Avaliação de Recursos Geotermais da Bacia do Paraná (Gomes, 2009) e de seu entorno, no qual foram realizadas perfilagens térmicas no Município de Montes Claros no ano de 2005.

Este projeto foi iniciado em 2012 pelo Laboratório de Geotermia da UFVJM - Universidade Federal dos Vales do Jequitinhonha e Mucuri, Campus do Mucuri em Teófilo Otoni, cujo laboratório se encontra em fase de implementação, com objetivo de obter-se um melhor entendimento do campo térmico local e regional.

As medidas dos gradientes geotérmicos foram realizadas pela equipe do Laboratório de Geotermia do Observatório Nacional/MCT do Rio de Janeiro.
Os dados utilizados para a avaliação e observação do gradiente geotérmico são integrantes de dados experimentais, medidos em poços de duas localidades, situadas dentro do Município de Montes Claros, onde um dos poços está localizado no bairro Jardim Primavera na região nordeste do município e o outro situado no bairro São Geraldo II na porção sul de Montes Claros (Gomes e Hamza, 2009; e Gomes, 2009).

\section{Geologia e Geofísica Regional}

$\mathrm{Na}$ área de Montes Claros o Cráton São Francisco é recoberto pela unidade neoproterozóica do Grupo Bambuí, que são parcialmente cobertos por sedimentos do cretáceo pertencentes à Formação Urucuia ou por cobertura detrito-lateríticas, coluviais e aluviais (CPRM, 2002).

Do ponto de vista geológico, o Município de Montes Claros pertence à Bacia do Alto Médio São Francisco, correspondendo à porção Sul do Cráton São Francisco sendo coberta por sedimentos de idades proterozóicas a fanerozóicas (CPRM, 2002).

Na Figura (1) apresentamos a localização do Município de Montes Claros, onde foram realizadas as medidas geotérmicas e situado na região Norte de Minas Gerais.

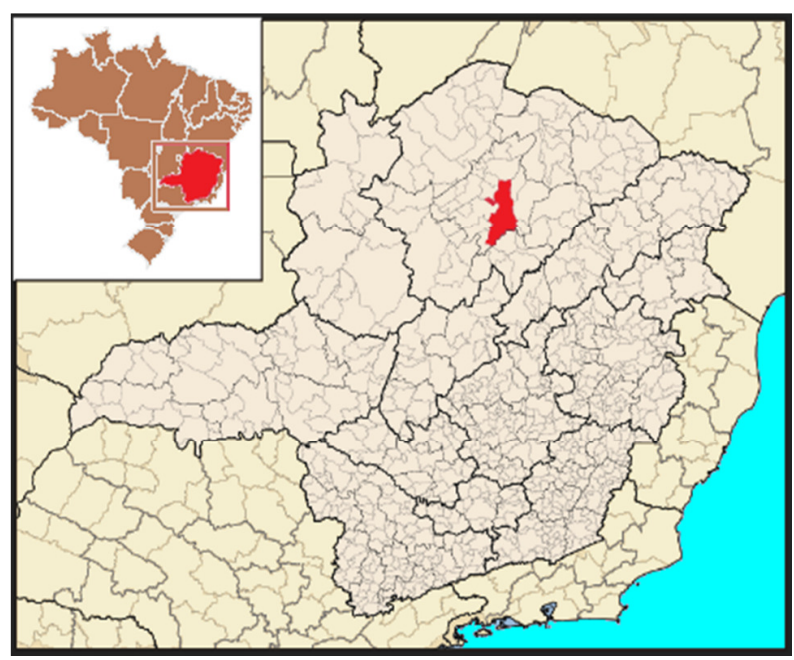

Figura 1- Mapa regional com a localização do Município de Montes Claros situado na região norte do Estado de Minas Gerais.

O grupo Bambuí é representado, basicamente, pelo Subgrupo Paraopeba indiviso e pela Formação Lagoa do Jacaré. O Subgrupo Paraopeba indiviso é formado, principalmente, por rochas pelíticas carbonatadas e sedimentos siliciclásticos. Já a formação Lagoa do 
Jacaré predomina os calcários, siltitos e margas (CPRM, 2002).

O Município está inserido na Formação Serra de Santa Helena, que constitui uma sucessão sedimentar predominantemente pelítica.

O clima do Município de Montes Claros é caracterizado como tropical, ou seja, com diminuição de chuvas no inverno, sendo estes secos e amenos, e verões chuvosos com altas temperaturas. (Oliveira et al., 2006).

\section{Metodologia}

Os métodos empregados para a determinação do gradiente geotérmico no presente trabalho foram 0 convencional (CVL) e o de temperatura estável do fundo de poço (CBT). Para a determinação do gradiente geotérmico no município de Montes Claros, foi utilizado somente o método de temperatura estável de fundo do poço (CBT).

O conhecimento das temperaturas em superfície é requisito básico para a avaliação de recursos geotermais e podem ser identificadas por medidas diretas e estimativas indiretas (Bolditzar, 1958; Bullard, 1965; Bodvarsson, 1974; Jessop et al, 1976; Haenel e Mongelli, 1988; Fournier, 1991).

Os dados obtidos são resultados de medições experimentais, nas quais foram utilizadas temperaturas relativamente antigas da área de estudo. Foi feita uma modificação nas temperaturas de superfície do trabalho realizado em Montes Claros por uma equipe do Laboratório de Geotermia do Observatório Nacional /MCT do Rio de Janeiro em 2005.

Foi utilizada uma temperatura mais estável do fundo do poço, tornando possível observar os valores de gradientes geotérmicos quando as perturbações não interferem tanto nas temperaturas. A temperatura utilizada foi de $24^{\circ} \mathrm{C}$.

As medidas de temperaturas foram efetuadas com o uso de sensores tipo termistor. Os termistores são constituídos por um material semicondutor cuja resistência elétrica diminui com 0 aumento da temperatura. Foi utilizado o termistor do tipo NTC (Negative Temperature Coeficiente), onde a resistência elétrica é inversamente proporcional à temperatura, sendo também um ótimo sensor onde seja necessária uma maior sensibilidade com as variações de temperaturas.

O método de temperatura estável de fundo do poço (CBT) é utilizado em casos que os fluxos de fluidos no interior do poço, perturbam o regime térmico condutivo, tornando impossível a determinação pelo método convencional do gradiente geotérmico.

Para isso fez-se necessária a adaptação de um método conhecido como método de fundo do poço (BHT), proposto por Carvalho e Vacquiers (1977) e Carvalho (1981), e foi utilizado por Hamza e Muñoz (1996), Gomes e Hamza, (2004, 2005, 2006, 2007, 2008 e 2009), Hamza et al, (2004), Hamza et al, (2005), Alexandrino e Hamza (2008), Hamza et al (2010), Gomes e Alexandrino (2011) com o escopo de que nesta situação seja possível determinar o gradiente térmico.
O gradiente geotérmico, pelo método CBT, utilizado no presente trabalho, foi determinado pela seguinte fórmula:

$$
\mathrm{T}_{\mathrm{CBT}}=\mathrm{T}_{0}+\mathrm{q} \sum_{\mathrm{i}=1}^{\mathrm{N}} \mathrm{R}_{\mathrm{i}} \mathrm{h}_{\mathrm{i}}
$$

onde $R_{i}$ é a resistividade térmica da camada $i$. O termo do somatório se refere à resistência térmica cumulativa das formações presentes até o fundo do poço onde foi efetuada a medida de temperatura.

\section{Resultados}

Os poços onde foram realizadas as perfilagens térmicas estão localizados no Município de Montes Claros e os mesmos estão corretamente georreferenciados como apresentado na Tabela (1) a seguir:

Tabela 1 - Local dos poços perfilados em Montes Claros (Gomes, 2009).

\begin{tabular}{|c|c|c|c|}
\hline \multirow{2}{*}{ Poço } & \multirow{2}{*}{ Local } & \multicolumn{2}{|c|}{$\begin{array}{c}\text { Coordenadas } \\
\text { Geográficas }\end{array}$} \\
\cline { 3 - 4 } & & Latitude & Longitude \\
\hline $\begin{array}{c}\text { MC01- } \\
\text { COPASA }\end{array}$ & $\begin{array}{c}\text { Jardim } \\
\text { Primavera }\end{array}$ & $16^{\circ} 41^{\prime} 34,3^{\prime \prime}$ & $43^{\circ} 48^{\prime} 51,1^{\prime \prime}$ \\
\hline $\begin{array}{c}\text { MC02- } \\
\text { COPASA }\end{array}$ & $\begin{array}{c}\text { São Geraldo } \\
\text { II }\end{array}$ & $16^{\circ} 47^{\prime} 29,8^{\prime \prime}$ & $43^{\circ} 53^{\prime} 13,6^{\prime \prime}$ \\
\hline
\end{tabular}

As perfilagens foram realizadas pela equipe do Laboratório de Geotermia do Observatório Nacional/MCT do Rio de Janeiro em trabalho de campo realizado no Município de Montes Claros, e na época foram consideradas as temperaturas de superfície no valor de $25{ }^{\circ} \mathrm{C}$ no poço $\mathrm{MC01}$, e de $25,25{ }^{\circ} \mathrm{C}$ no poço $\mathrm{MC02}$, ambas as análises realizadas com método CBT.

Qualquer alteração no valor da temperatura de superfície, faz com que, nas mesmas perfilagens térmicas, mudem abruptamente os valores dos gradientes geotérmicos.

Foi utilizada uma temperatura de superfície ajustada com base na região estável do fundo do poço, no caso $24^{\circ} \mathrm{C}$, que se aproxima mais das observações dos ajustes lineares com o método CVL.

Não encontramos na literatura registros de temperatura anteriores à década de 70 , motivo pelo qual escolhemos a temperatura resultante do ajuste linear dos dados experimentais.

Os resultados obtidos de gradientes geotérmicos em ambos os poços, quando modificamos a temperatura de subsuperfície, são apresentados na Tabela (2).

Os perfis térmicos com os valores da temperatura medidos durante as perfilagens térmicas, integrantes do conjunto de dados utilizados na determinação do gradiente geotérmico de cada poço, são apresentados graficamente no final deste trabalho nas Figuras (2) e (3). Os novos valores obtidos do gradiente geotérmico apresentaram resultados compreendidos no intervalo situado entre 18,6 e $20,2^{\circ} \mathrm{C} / \mathrm{km}$. 
Tabela 2- Resultados obtidos dos gradientes geotérmicos considerando temperaturas de superfície antigas (Serra, 1955).

\begin{tabular}{|c|c|c|c|}
\hline \multirow{2}{*}{ Poço } & $\begin{array}{c}\text { Temperatura } \\
\left({ }^{\circ} \mathbf{C}\right)\end{array}$ & \multicolumn{2}{|c|}{$\begin{array}{c}\text { Gradiente } \\
\text { Geotérmico } \\
\left({ }^{\circ} \mathbf{C} / \mathbf{k m}\right)\end{array}$} \\
\cline { 3 - 4 } & & Tipo & Valor \\
\hline $\begin{array}{c}\text { MC01- } \\
\text { COPASA }\end{array}$ & 24,000 & CBT & 20,2 \\
\hline $\begin{array}{c}\text { MC02- } \\
\text { COPASA }\end{array}$ & 24,000 & CBT & 18,6 \\
\hline
\end{tabular}

\section{Conclusões}

O valor médio obtido para o gradiente geotérmico do Município de Montes Claros foi de 19,4 $\pm 0,13^{\circ} \mathrm{C} / \mathrm{km}$. O gradiente geotérmico no poço MC01, sendo a temperatura da superfície $25^{\circ} \mathrm{C}$, foi de $9,3{ }^{\circ} \mathrm{C} / \mathrm{km}$ e quando diminuímos a temperatura da superfície para $24^{\circ} \mathrm{C}$ o gradiente passa para $20,2{ }^{\circ} \mathrm{C} / \mathrm{km}$, ou seja, uma diminuição de $1^{\circ} \mathrm{C}$ na temperatura da superfície resultou em um aumento de $10,87{ }^{\circ} \mathrm{C} / \mathrm{km}$ no gradiente geotérmico.

No poço MC02, quando modificamos a temperatura de superfície para $24^{\circ} \mathrm{C}$ observamos uma variação no gradiente geotérmico de $12,3^{\circ} \mathrm{C} / \mathrm{km}$.

Os resultados dos gradientes geotérmicos obtidos pelo método CBT nos poços localizados em Montes Claros, utilizando temperaturas de superfície ajustadas em profundidades entre 80 e $100 \mathrm{~m}$, livres de acentuadas perturbações do fundo do poço.

Os novos valores indicaram que os gradientes geotérmicos se encontram num intervalo compreendido entre 18,6 e $20,2^{\circ} \mathrm{C} / \mathrm{km}$.

Os novos resultados são indicativos de regiões précambrianas compreendidas por bacias sedimentares.

\section{Agradecimentos}

Agradecemos ao Dr. Prof. Valiya Mannathal Hamza e a toda a equipe do Laboratório de Geotermia do Observatório Nacional/MCT do Rio de Janeiro, que realizou as perfilagens e à FAPEMIG pela bolsa de iniciação científica.

\section{Referências}

Bolditzar, 1958 T. Bolditzar, The distribution of temperatures in flowing wells, Am. J. Sci. 256 (1958), pp. 294-298.
CPRM - Companhia de Pesquisa de Recursos Minerais 2002. Projeto São Francisco: Caracterização Hidrogeológica da Micro Região de Montes Claros. Angélica G. Soares, Eduardo J.M. Simões, Ely S. de Oliveira, Haroldo S. Viana - Belo Horizonte: SEME/COMIG/CPRM, 2002.

Fournier, R. O., 1991, Water geothermometers applied to geothermal energy, ed. D'Amore F., UNITAR-UNDP, New York, 253-273.

Gomes, A.J.L., Hamza, V.M., 2003, Avaliação de Recursos Geotermais do Estado do Rio de Janeiro, $8^{\circ}$ International Congress of the Brazilian Geophysical Society, Rio de Janeiro.

Gomes, A. J. L. and HAMZA, V.M., 2005. Gradiente e Fluxo Geotérmico do Estado de Santa Catarina, 9。 International Congress of the Brazilian Geophysical Society, 6p., Cd-rom, Salvador, Brasil.

Gomes, A.J.L. e Hamza, V.M., 2006. Gradiente e Fluxo Geotérmico no Estado do Paraná, In: $2^{\circ}$ Congresso Internacional da SBGf, Anais, Cd-rom, Natal, 21-23 de setembro, 6p.

Gomes, A.J.L. e Hamza, V.M., 2007. Gradiente e Fluxo Geotérmico nas Bordas Norte e Noroeste da Bacia do Paraná, In: $10^{\circ}$ Congresso Internacional da SBGf, Anais, Cd-rom, Rio de Janeiro, 19-22 de Novembro, 6p.

Gomes, A.J.L. e Hamza, V.M., 2009. Gradiente e Fluxo Geotérmico da Bacia do Paraná, 11th International Congress of the Brazilian Geophysical Society held in August 24-28, 2009, Salvador, Brazil.

Hamza, V.M. and Muñoz, M., 1996. Heat Flow map of South America, Geothermics, V.I 25, n ${ }^{\circ} 6$, pp. 599-646.

Hamza, V.M., Silva Dias, F.J.S., Gomes, A.J.L. and Terceros, Z.G.D., 2005. Numerical and Functional Representations of Regional Heat Flow in South America, Physics of the Earth and Planet Int., V152, 4, p.223-256.

Meister, E.M., 1973. Gradientes geotérmicos nas bacias sedimentares Brasileiras, Boletim Técnico Petrobrás, 16 (4), 221-232.

Chaves, M.L.S.C. et al. Geologia integrada das Folhas Jequitaí, Bocaiúva e Montes Claros, norte de Minas Gerais, 20 de Outubro 2011.

Oliveira, I.M. et al., 2010. Utilizando as novas tecnologias visando o mapeamento do uso do solo do bairro Camilo Prates e seu entorno, $1^{\circ}$ Congresso Brasileiro de Organização de Espaço e $5^{\circ}$ Seminário de PósGraduação em Geografia da Unesp/Rio Claro, Rio Claro/SP, 5-7 de Outubro, 13p. 


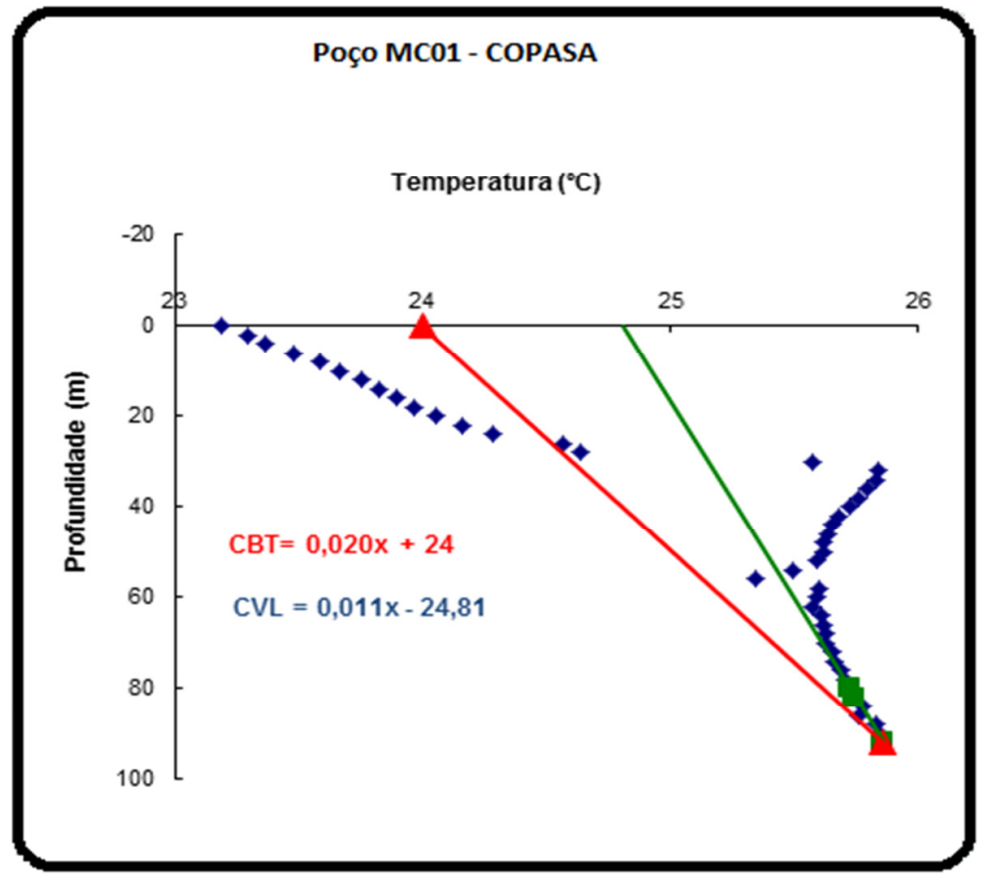

Figura 2 - Perfil térmico do poço MC01 da COPASA situado no bairro Jardim Primavera em Montes Claros com gradientes $C B T$ e $C V L$.

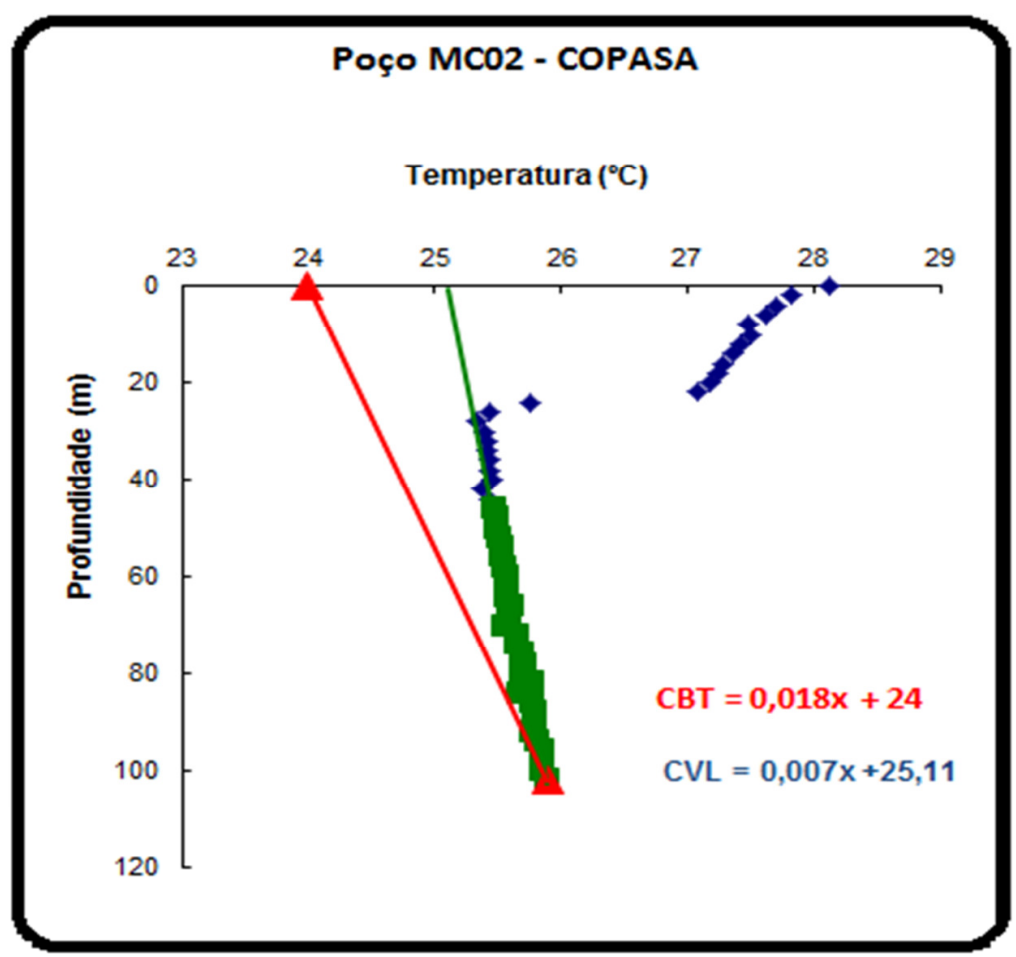

Figura 3 - Perfil térmico do poço MCO2 da COPASA situado no bairro São Geraldo Il em Montes Claros com gradientes CBT e CVL. 